\section{Logical foundations of computation}

The Theory of Computer Science: 4 Programming Approach. By J. M. Brady. Pp. xiii+276. (Wiley: New York and London, 1977.) Hardback £8; paperback $£ 4.50$.

BRADY has written a book which can be warmly recommended to readers wishing to understand the logical foundations on which computation rests. It will be particularly valuable to students of computer science, but may be of interest also to computer programmers who wish to widen their horizons.

As a practical discipline, computing grew up in the late 1950 s and early 1960s. Recruits to the programming profession came from mathematics, engineering, science, and even the classics and humanities. These people applied a largely intuitive approach to solving problems pressed upon them by commercial and other users, urgently requiring effective solutions. There were many difficulties, and many projects were late in completion, cost more than was intended, or were even aborted before completion. The lessons learned during this period led to the formulation of a set of methodologies for program design and construction which are now often graced with the name "Software Engineering".

To aid the newly emerging discipline, theoretical foundations have been and are being developed. Methods of defining the syntax and semantics of programming languages, methods of proving the correctness and equivalence of programs, and of analysing the complexity of algorithms, are theoretical developments with immediate potential impact.

Arising, however, from the mathematics and logic camp there has come a theoretical background to computing which is often criticised as quite irrelevant to the practising programmer. Brady argues that this is due to a misunderstanding of its position in the logical structure of the subject. The theories of computability, based on Abstract (Turing) Machines and General Recursive Functions belong to the meta-theory the goals of which are to define precisely the meaning of 'computable' and to discover the theoretical limits of computability.

His book is accordingly divided into two parts. Part 1 covers the metatheory, whereas part 2 (which has a different title in the contents list and in the text) discusses the approach to a theory of computer science.

Brady does not assume extensive mathematical knowledge of his readers, and the prerequisites are summarised in an appendix. Throughout the book he leans heavily on the intuitions of the reader as an experienced programmer. He compares his approach with that of M. L. Minsky (Computation: Finite and Infinite Machines, Prentice-Hall 1967) by suggesting that his book is to programming what Minsky's is to hardware; hence the second part of his title. He writes with an enthusiastic style which makes his text very readable. There are frequent invitations to the reader to extend his understanding by proving riders and solving problems. These he calls "checkpoints", a usage which I find irritating. There are also more typographical errors than one expects in a book of this quality. These are, however, minor blemishes in an otherwise excellent book.

\section{S. J. Goldsack}

S. J. Goldsack is Professor of Computing Science at Imperial College of Science and Technology, University of London, UK.

\section{Neutrino}

\section{proceedings}

Proceedings of the International Neutrino Conference, Aachen 1976. Edited by H. Faissner, H. Reithler and P. Zerwas. Pp. xii +748 . (Vieweg : Braunschweig, 1977.) DM168.

IT has been interesting to review this conference report while attending the 1977 Lepton-Photon Symposium in Hamburg, which covers the same field and much more. Rather than making last year's news seem old and dull, this year's conference illustrates how useful the Aachen proceedings can be as a reference. Aachen was a specialised conference and the speakers had time to do their experiments and theories justice. At conferences with a wider spread of subject matter the resulits can be reported but the details are lost.

Some of the results reported at Aachen have, of course, now been overtaken by better experiments, or modified by more careful analysis. Nevertheless, much of the picture is the same as it was last year. The fourquark theory, with charm, still stands and a great deal of the evidence for this is reported in these proceedings; including a few wisely chosen nonneutrino results from $\mathrm{e}^{+} \mathrm{e}^{-}$storage rings. The quark-parton picture of hadrons is being increasingly refined, with about the correct amount of scalebreaking to satisfy the asymptotically free gauge theories. As well as data on these effects there is a useful review by the late Benjamin Lee, who includes a simple introduction to scale-breaking in the course of his final summary of the whole conference.

Theoretical contributions from Dalitz, Cabibbo, Salam and others give a picture of the variety of different quark models still available. M. K. Gaillard and de Rujula discuss the success of the charm concept in the light of the rich spectrum of particles reported in the experimental sessions. In contrast to their confidence, there are calls from Wolfenstein, from Fritsch and from Sakurai for more data on neutral currents, on second class weak currents, on neutrino oscillations, on parity violation in atomic physics, and so on. We certainly do not yet understand the weak interaction completely.

As well as high energy accelerator physics, there are a few papers on neutrino astrophysics and on assorted low-energy topics such as double $\beta$ decay, and the ${ }^{3} \mathrm{H}$ spectrum as a probe of the neutrino mass.

David J. Miller

David Miller is Lecturer in Physics at

University College, London, UK.
BOOKS

ON PURE

AND APPLIED SCIENCE

Books reviewed or men-

tioned in this journal are available from stock.

Catalogues on application.

Please state interests.

\section{SCIENTIFIC LIBRARY}

ANNUAL SUBSCRIPTION from $£ 7.00$

Reduced rates for multiple subscriptions Available in U.K. only

Prospectus free on request

H. K. LEWIS \& Co. Ltd.

LONDON: 136 GOWER STREET, WC1E 6BS

Telephone: $01-3874282$ 